\title{
Interpretación de la Constitución y juez constitucional*
}

\author{
Interpretation of the constitution \\ and constitutional judge
}

\section{Francisco Javier Díaz Revorio**}

\section{RESUMEN}

La interpretación de la Constitución es una tarea compleja y llena de dificultades, relativas al "cómo" y al "quién". Se trata de un proceso que comparte algunas caracteristicas con la interpretación de cualquier otra norma, pero que reviste también notorias peculiaridades. Éstas derivan, en buena medida, de las características de la propia norma constitucional; en especial su generalidad, ambigüedad, politicidad y carácter axiológico, asi como de la notable presencia de principios en la norma fundamental. Por ello, sin perjuicio de la aplicabilidad a la Constitución de los criterios o elementos habitualmente utilizados en la interpretación juridica, se han propuestos diversos métodos específicos para la interpretación constitucional, como la concretización, el método tópico, o la ponderación. Pero todos ellos presentan alguna carencia cuando se les quiere utilizar como un método global y universal de interpretación constitucional.

\section{ABSTRACT}

The interpretation of the Constitution is a complex task with difficulties, concerning both the "how" and the "who". It is a process that shares some characteristics with the interpretation of any other norm, but also has notorious peculiarities. These derive largely from characteristics of constitutional provision; especially its generality, ambiguity, politicity and axiological character; as well as the notable presence of principles in the fundamental rule.

Therefore, without prejudice to the applicability of criteria or elements commonly used in legal interpretation to the Constitution, various specific methods for constitutional interpretation have been proposed, such as "concretization", topical method, or weighting.

But they all pose inadequacies, when they are used as global and universal methods of constitutional interpretation. As for the sub-

\footnotetext{
* Recibido: 13 de julio de 2015. Aceptado: 17 de agosto de 2015.

** Catedrático de Derecho Constitucional en la Universidad de Castilla-La Mancha, Toledo, España. (cojavier.drevorio@uclm.es)
} 
En cuanto a los sujetos, se sostiene que, privados o públicos, pueden realizar esa labor, pero están en una posición especial el legislador y el juez; en especial el juez constitucional. El trabajo se centra en el análisis de la posición especial del Tribunal Constitucional o la jurisdicción constitucional especifica equivalente como intérprete supremo de la Constitución. En esta condición, sus decisiones tienen un valor especial, vinculante para todos los jueces y tribunales, e incluso en algunos casos poseen efectos erga omnes o derogatorios de las leyes. Eso es lo que da a la jurisprudencia constitucional el valor de una fuente del derecho, y al Tribunal Constitucional, una posición de poder de gran relevancia, en la cual puede enfrentar a los otros intérpretes constitucionales. En su condición de delegado del poder constituyente, su poder es grande, aunque sobre el mismo, todo sistema ha de establecer algunos controles.

PALABRAS ClAVE: Constitución, Tribunal Constitucional, Justicia Constitucional, jurisdicción constitucional, interpretación constitucional, Poder Judicial, juez constitucional. jects, it is argued that all subjects, private or public, can perform this work, but legislators and judges are in a special position; especially the constitutional judge.

This work focuses in the analysis the special position of the Constitutional Court (or the specific constitutional jurisdiction equivalent) as the supreme interpreter of the Constitution. In this condition, its decisions have a special value, binding for all judges and courts, and even in some cases have "erga omnes" effects or derogatory laws.

This is what gives constitutional jurisprudence the value of a source of law, and to the Constitutional Court a powerful position of great relevance; in that it can confront other constitutional interpreters. In its capacity as the delegate of constituent power, its power is great, although some controls over the whole system have to be established.

KEY WORDS: Constitution, constitutional Law, constitutional interpretation, Constitutional Court, constitutional judge, Judicial Power, constitutional justice, constitutional jurisdiction.

\section{SUMARIO}

1. Planteamiento

2. Interpretación juridica e interpretación constitucional

3. A la búsqueda de un método para la interpretación constitucional

4. Los intérpretes de la Constitución

5. El Tribunal Constitucional y la interpretación de la Constitución

6. El valor de la jurisprudencia constitucional 


\section{Planteamiento}

La interpretación de la Constitución, así como la interpretación constitucional de la ley, representa uno de los problemas más complejos, pero también más acuciantes, de la teoría y la práctica constitucionales. La Constitución es la norma suprema de una comunidad; buena parte de sus preceptos se caracterizan por su generalidad y ambigüedad, por lo cual las cuestiones del cómo y el quién ha de asumir la labor de interpretación adquieren una relevancia fundamental. En esta época, las casi eternas cuestiones que se plantean, lejos de ir solventándose, permanecen abiertas, lo cual representa un reto permanente y puede llegar a resultar dramático.

En lo relativo al cómo, parece cada vez más evidente que la labor de interpretación constitucional adquiere unos perfiles netamente específicos, que conllevan la necesidad de buscar un método también propio. Pero también es preciso determinar en qué medida ese método debe mantener, prescindir o adaptar los métodos y elementos usados habitualmente en la interpretación de la ley. En cualquier caso, creo que los diversos intentos de establecer un método específico para la interpretación constitucional plantean diversas limitaciones y carencias. Así, contemplan la norma fundamental y su aplicación desde perspectivas parciales o unidimensionales, aunque probablemente la ponderación pasa a ser, entre los diversos métodos utilizados, el que más éxito ha llegado a tener.

Por otro lado, la cuestión relativa al quién se desglosa en dos aspectos. En primer lugar, quién puede o debe interpretar la Constitución; en segundo término, quién tiene la última palabra en materia de interpretación constitucional. La primera requiere, a mi juicio, una respuesta lo más abierta posible. La segunda encierra un reto permanente y siempre pendiente de una respuesta justificada en términos de legitimidad. En efecto, cuando se atribuye el carácter de intérprete supremo de la Constitución al Tribunal Constitucional, se está planteando la cuestión de su legitimidad frente al juez, en lo relativo a la interpretación constitucional de la ley, sobre todo frente al legislador democrático. La cuestión de quién tiene la última palabra en la interpretación de la Constitución es, en el fondo, una cuestión de poder y de legitimidad. Quien asuma esa posición tiende a situarse en el mismo nivel del poder constituyente, del cual, en cierto modo, actúa como "delegado". De ahí la importancia de fijar los límites de la función suprema de interpretación de la Constitución.

Resulta imposible un análisis que resuelva tan trascendentales retos; sin embargo, intentaré aportar alguna reflexión que ayude a establecer las pautas para el ejercicio de esa labor. Mantendré una perspectiva neutral y tan científica como sea posible, aunque desde una óptica personal. 


\section{Interpretación jurídica e interpretación constitucional}

El punto de partida será precisar en qué medida la interpretación de la Constitución es una labor diferente a la interpretación jurídica en general. En tal caso, si es necesaria, y posible, la aplicación de un método específico.

La Constitución es normalmente un texto escrito, y como tal, está compuesta de un conjunto de oraciones formadas por palabras que expresan algo. Cualquier lector que se acerque al texto constitucional tratará de extraer el sentido y significado que se expresa en esas palabras. Como todo texto, la Constitución es susceptible de interpretación. Siguiendo a Zagrebelsky, podemos entender interpretación como "el proceso intelectivo a través del cual, partiendo de las fórmulas lingüísticas que forman un enunciado, se llega a un contenido, es decir, se pasa de los significantes a los significados". ${ }^{1}$ Si bien el término interpretación puede referirse tanto a este proceso como al resultado del mismo.

La Constitución es un texto jurídico; su interpretación participa por ello de las características propias de la interpretación del derecho. Desde luego, la interpretación jurídica comparte con cualquier proceso interpretativo la finalidad de descifrar el significado de textos lingüísticos, pero los textos jurídicos contienen normas. Así, el significado que se trata de extraer del enunciado lingüístico es una norma jurídica, entendida como "mandato o prohibición dirigido a poderes públicos o a ciudadanos, y cuyo incumplimiento es objeto de una respuesta mediante una sanción jurídica". ${ }^{2}$ Esta especificidad de la interpretación jurídica no sólo afecta al objeto de la misma, sino que también condiciona el propio proceso interpretativo.

En la interpretación jurídica el significante es la disposición, y el significado es la norma. De esta forma, resulta esencial la distinción, apuntada entre otros por Guastini, ${ }^{3}$ entre disposición, entendida como cualquier enunciado que forma parte de un documento normativo del discurso de las fuentes, y norma, es decir, cualquier enunciado que constituya el sentido o significado adscrito a una o varias disposiciones o fragmentos de ellas.

Interpretar un texto jurídico es, por tanto, atribuirle un sentido o significado normativo, hallar o descifrar la norma que deriva del mismo. No hay norma

\footnotetext{
1 Zagrebelsky, Gustavo. "La Corte constitucional y la interpretación de la Constitución", en Antonio López Pina (Ed.), División de poderes e interpretación, Tecnos, Madrid, 1987, p. 161.

${ }^{2}$ Naturalmente, es posible encontrar en los textos legales o constitucionales preceptos aislados que aparentemente no responden a este esquema de mandato o prohibición con respuesta jurídica, pero normalmente esta respuesta viene dada por la pertenencia de los propios preceptos al ordenamiento jurídico. Por ello, el carácter jurídico de la norma deriva de la pertenencia a este ordenamiento considerado como sistema de reglas, normas e instituciones jurídicamente sancionadas.

${ }^{3}$ Guastinı, Riccardo. Le fonte del diritto e l'interpretazione, Giuffrè, Milán, 1993, p. 118.
} 
sin previa actividad interpretativa, ni disposición para referirse al resultado del proceso interpretativo. Desde la perspectiva de este proceso, las disposiciones constituyen su objeto, y las normas su resultado.

En las líneas anteriores he utilizado términos como atribuir y hallar. Sin embargo, cada uno de ellos refleja una concepción diferente del proceso interpretativo. Entre otros, Wrobleski señala una de las más relevantes cuestiones relativas al proceso de interpretación jurídica: si es que viene encaminado a descubrir la norma preexistente, derivada del enunciado normativo, o si trata de atribuir a dicho enunciado un significado normativo. ${ }^{4}$ Se trata de saber si el proceso interpretativo es o no creativo.

La cuestión reviste una gran complejidad, pero cabe apuntar que, salvo en los inusuales casos donde el sentido del precepto jurídico aparece como único, en todo proceso interpretativo hay una cierta labor creadora, que implicará creación de nuevo Derecho, en la medida en que el propio ordenamiento atribuya al intérprete la potestad de adoptar decisiones vinculantes para terceros. Así sucede en el caso de que dicha interpretación venga realizada por los tribunales, o al menos por aquellos que se sitúan en la cúspide del sistema judicial.

Hay quien afırma que, en sentido estricto, sólo existe interpretación cuando hay realmente duda en el sentido que ha de atribuírsele a un enunciado normativo, según el clásico aforismo in claris non fit interpretatio. Estos casos "claros" son en la práctica una minoría; hay una evidente dificultad para distinguirlos de los que no lo son. En la mayoría de ocasiones, la labor interpretativa conlleva cierta creación, ya que del enunciado legal no siempre se deduce de forma indubitada un único contenido normativo o una única respuesta a un caso concreto. Por todo ello, cabe utilizar el término interpretación en un sentido amplio al incluir también los casos "claros", o en el sentido estricto, referido sólo a los supuestos dudosos.

La Constitución es una norma peculiar desde varios puntos de vista por su rango, contenido y finalidad. Estas peculiaridades afectan su método de interpretación, el cual, en palabras de Canosa Usera, ha de plegarse a su objeto. ${ }^{5}$ La cuestión de si estas especialidades vienen a configurar un proceso cualitativamente distinto, o sólo una diferencia de grado respecto al método de interpretación jurídica en general, es difícil de responder categóricamente.

Conviene precisar, en todo caso, que los elementos y criterios propios de la interpretación jurídica son aplicables a la interpretación constitucional, si bien con algunos matices. No obstante, resultan insuficientes y deben ser

\footnotetext{
${ }^{4}$ Wrobleski, JeRzy. Constitución y teoría general de la interpretación jurídica, Cuadernos Civitas, Madrid, 1985, p. 17. ${ }^{5}$ Canosa Usera, Raúl. Interpretación constitucional y fórmula politica, Centro de Estudios Constitucionales, Madrid, 1988.
} 
completados con otros criterios propios. Por ello estimo que la interpretación constitucional presenta diferencias muy apreciables con el resto de la interpretación jurídica, aun partiendo de su misma base y participando de sus elementos. Se trata de un proceso con acusadas especialidades, pero no de un proceso esencialmente distinto.

Sin embargo, más que la ubicación de este proceso en una u otra categoría, es importante la precisión de cuáles son esas especialidades que afectan al objeto de la interpretación, el método de la misma y los sujetos que tienen encomendada esta labor.

En primer lugar, hay que destacar las notas peculiares que caracterizan al texto constitucional, ya que en buena medida las especialidades de la interpretación constitucional derivan de su objeto. Como ha enfatizado por ejemplo Pérez Royo, ${ }^{6}$ la Constitución posee una estructura normativa diferente a la ley; a diferencia de ésta, la Constitución es una norma única, que no es expresión de regularidad en los comportamientos sociales. Además, su estructura no supone habitualmente la tipificación de una conducta para anudarle determinadas consecuencias jurídicas.

En general, la Constitución es el cauce, el marco, el límite a la actuación de particulares y poderes públicos; en especial del legislador. Sin embargo, tampoco esta idea puede afirmarse de forma categórica, pues también la Constitución contiene mandatos de actuación positiva para los poderes públicos. De este modo, estos poderes públicos, en primer término el Poder Legislativo, actuar con el límite de la Constitución, aunque en ciertos casos también deberán desarrollarla.

La auténtica especialidad de la interpretación constitucional consiste en que "los mandatos constitucionales son susceptibles de desarrollo infinito, tanto por el juez como por el legislador, y ambos pueden llegar a soluciones divergentes". ${ }^{7}$ Con todo, todavía es posible, y aun necesario, deslindar la posición de uno y otro en relación con la Constitución.

Ahora interesa destacar algunos de los elementos esenciales en los que se traduce la especial estructura normativa de la norma fundamental como objeto de la interpretación constitucional. En primer lugar, puede destacarse el carácter abierto de muchos de sus preceptos, los cuales suelen reflejar cierta ambigüedad. En ocasiones, esta ambigüedad se busca intencionalmente; a veces, los preceptos constitucionales son "fórmulas de compromiso que son precisamente expresión de la falta de acuerdo y que postergan la decisión”. ${ }^{8}$

\footnotetext{
${ }_{6}^{6}$ Pérez Royo, Javier. Curso de Derecho Constitucional, Marcial Pons, Madrid, 2000, p. 135.

${ }^{7}$ Alonso Garcia, Enrioue. La interpretación de la Constitución, Centro de Estudios Constitucionales, Madrid, 1984, p. 26.

${ }^{8}$ BöcKenförde, ERnst Wolfgang. "Los métodos de interpretación constitucional. Inventario y crítica" (1976), en Escritos
} 
Pero el empleo de cláusulas generales y declaraciones de principio es otras veces consustancial a la naturaleza de los conceptos, ideas y valores que acoge la norma fundamental. Desde luego, el empleo de fórmulas abiertas no es una nota exclusiva del texto constitucional, ya que existen conceptos jurídicos indeterminados en todos los sectores del ordenamiento; pero la frecuencia de estos conceptos en la norma constitucional es más acusada que en cualquier otra norma jurídica.

Una segunda nota relevante de la norma constitucional es la politicidad, entendida como la conexión con los diversos sujetos, grupos y órganos que manifiestan y expresan el poder del Estado, y que están presentes tanto en la elaboración de la Constitución como en su desarrollo e interpretación. La realización de la norma fundamental, llevada a cabo por el legislador o el Gobierno, posee un componente político incuestionable. De este modo, la interpretación constitucional decide sobre problemas políticamente relevantes en mayor medida que en la interpretación de otras normas jurídicas.

Desde luego, el intérprete judicial de la Constitución, en particular el Tribunal Constitucional, debe decidir las controversias constitucionales con criterios jurídicos, aunque también debe ser consciente de las consecuencias políticas de sus decisiones. Por tanto, este carácter político tiende a expandirse al método y al intérprete. Aunque, como afirma Leibholz, ${ }^{9}$ el Tribunal Constitucional resuelve "conflictos jurídicos sobre materia política", porque el carácter político de un acto no impide el conocimiento jurídico del mismo. ${ }^{10}$ Este es, sin duda, uno de los retos fundamentales del intérprete constitucional: dar una respuesta jurídica a un problema político. Es un reto inmenso, pero en cuya superación debería seguir confiando todo jurista.

Entre otras características de la norma constitucional, está el carácter axiológico, ya que el texto fundamental contiene valores y principios generales, tales como libertad, igualdad, justicia, dignidad, Estado social y democrático de derecho. Éstos suponen límites, mandatos y fines que el poder constituyente impone a los poderes públicos. No cabe aquí extenderse en la distinción entre valores y principios, ${ }^{11}$ pero la Constitución tiene en general una textura principal. En la misma, predominan los principios, entendidos en sentido amplio (incluyendo valores), sobre las reglas.

sobre Derechos Fundamentales, Nomos Verlagsgesellschaft, Baden-Baden, 1993, p. 17.

${ }^{9}$ LeibHolz, GeRHARD. "El Tribunal Constitucional de la RFa y el problema de apreciación judicial de la política", en Gerhard Leibholz, Problemas fundamentales de la Democracia moderna, Instituto de Estudios Políticos, Madrid, 1971, p. 149.

${ }^{10}$ BaСноF, Отто. Jueces y Constitución, Civitas, Madrid, 1989, p. 61.

${ }^{11}$ Sobre este punto, Diaz Revorio, Francisco Javier. Valores superiores e interpretación constitucional, Centro de Estudios Políticos y Constitucionales, Madrid, 1997, p. 97. 
Esta característica está vinculada con las dos anteriores y, en alguna medida, es causa de las mismas. Así, condiciona el método de la interpretación constitucional, planteando dificultades interpretativas. En relación con la interpretación, estos contenidos axiológicos juegan un doble papel. Por un lado, son ellos mismos objeto de la interpretación de los diversos poderes públicos, quienes deben tener en cuenta su carácter general y abierto, así como su contenido o significado ineludible. Por otro lado, estos valores y principios son también elemento o instrumento para la interpretación del resto de la norma constitucional y del ordenamiento jurídico en su conjunto. Desde esta perspectiva, presiden todo criterio o método interpretativo y dotan al sistema jurídico de unidad y coherencia.

Todas las particularidades del objeto condicionan el método de la interpretación y la posición de los distintos intérpretes. En efecto, el legislador es el primer sujeto cualificado para precisar el contenido de estas cláusulas abiertas, desarrollarlas y completar su significado. Mientras, el Tribunal Constitucional debe señalar, en principio, cuándo el Poder Legislativo traspasa los límites del texto constitucional en su interpretación o desarrollo. Esta circunstancia debe presentarse en el método de la interpretación constitucional, pero no debe entenderse ésta de forma absoluta. La Constitución contiene también preceptos más precisos, así como mandatos de actuación que poseen cierto grado de concreción. Ciertamente, caben diversas lecturas de la Constitución, y el legislador es el primer sujeto legitimado para optar por una u otra. Pero no cabe cualquier lectura de la norma fundamental, pues estas notas no implican ausencia de contenido normativo o de carácter vinculante en ella.

\section{A la búsqueda de un método para la interpretación constitucional}

Como he señalado, las peculiaridades del objeto de la interpretación constitucional penetran de forma inevitable en el método de la misma. Ello no significa que en la interpretación constitucional no sean útiles los elementos o argumentos tradicionalmente empleados en la interpretación jurídica. De hecho, son habitualmente utilizados por los tribunales constitucionales y por los tribunales ordinarios en su interpretación de la Constitución. Aunque presentan sus propias peculiaridades cuando se utilizan para interpretar la norma fundamental. De esta manera, no actúan de idéntica forma a como lo hacen generalmente en la interpretación de normas infraconstitucionales. Pero, por otro lado, se muestran insuficientes en esta labor, de manera que han de ser completados con otros criterios o elementos propios de la interpretación constitucional. 
La cuestión es si la conjunción de estos diversos criterios y elementos (los comunes y los específicos) configura realmente un método de la interpretación constitucional, o si éste debe estar regido por una característica y un modo de actuar único. Antes de aproximarme a este punto, conviene que nos detengamos en cada uno de los criterios tradicionales, en su aplicación a la interpretación constitucional. Como es sabido, estos criterios o reglas fueron formulados por Savigny ${ }^{12}$ y suelen recogerse, con alguna modificación, en la mayoría de los códigos civiles.

Criterio gramatical. Se basa en el sentido propio de las palabras. Esto es, en la dicción literal del texto. Es un imprescindible punto de partida en toda interpretación jurídica, por tanto, también en la interpretación constitucional. En aquellos supuestos en que la dicción literal es clara y terminante, la interpretación gramatical es el método más fiable. Así, por ejemplo, el propio Tribunal Constitucional español ha afirmado que "una interpretación que conduzca a un resultado distinto de la literalidad del texto [sólo puede pensarse] cuando existe ambigüedad o cuando la ambigüedad puede derivar de conexión o coherencia sistemática entre preceptos constitucionales". ${ }^{13} \mathrm{Sin}$ embargo, y tomando en cuenta la frecuencia de términos generales y ambiguos en la redacción de la Constitución, el criterio literal o gramatical se muestra manifiestamente insuficiente en esta labor interpretativa, y sólo en contados casos puede resultar decisivo.

Criterio sistemático. Este elemento posee una gran importancia en la interpretación constitucional. Entendido en sentido amplio, engloba tres argumentos. El argumento a coherentia, según el cual los enunciados legales han de interpretarse tomando en cuenta que no pueden expresar normas incompatibles. El argumento sedes materiae, por el cual se atribuye un significado normativo a un precepto dudoso tomando en cuenta el lugar que ocupa el texto normativo del que forma parte. Por último, el argumento sistemático en sentido estricto, que atribuye un significado normativo a un enunciado tomando en cuenta otros preceptos o normas. Esto es, al contexto jurídico al que se refiere, por ejemplo, el artículo 3.1 del Código Civil español. Todos ellos presentan notable utilidad a la hora de interpretar la Constitución y son utilizados reiteradamente por los tribunales constitucionales. ${ }^{14}$

\footnotetext{
${ }^{12}$ Savigny, Friedrich Karl Von. Sistema de Derecho romano actual, vol. 2, [en línea] Sirio. [Citado: 27. Febrero. 2016]. Disponible en: http://sirio.ua.es/libros/BDerecho/Sistema_derecho_romano_actual_1_y_2/index.htm

${ }^{13}$ STC $72 / 1984$, de 14 de junio, f. j. 6.

${ }^{14}$ Siguiendo por ejemplo al Tribunal Constitucional español, a título de simple muestra, cabe señalar que ha utilizado el argumento a coherentia para interpretar la prerrogativa de la inmunidad parlamentaria de forma coherente y no contradictoria con los valores igualdad y justicia y el derecho a la tutela judicial efectiva (por ejemplo, sstc 90/1985, de 22 de julio, o 206/1992, de 27 de noviembre, f. j. 3, sentencias que, al tiempo, realizan una interpretación finalista
} 
Los antecedentes históricos y legislativos. En el caso de la norma fundamental, nos referimos a los precedentes constitucionales y los debates parlamentarios que dieron origen a su aprobación. Este elemento también ha sido utilizado por el Tribunal Constitucional (por ejemplo, ssTc 27/1.981, de 20 de julio, f. j. 2; 67/1.983, de 22 de julio, f. j. 3; 53/1.985, de 11 de abril), pero su importancia es relativa. Más bien, debe entenderse como un argumento de apoyo a otros, que rara vez resultará decisivo si se utiliza de forma aislada y, desde luego, no parece que pueda prevalecer sobre los restantes cuando estos apuntan a otra interpretación. El propio Tribunal Constitucional español ha afirmado que los debates parlamentarios "son un importante elemento de interpretación, aunque no la determinen". ${ }^{15}$

Interpretación teleológica. Es la que busca el significado de un precepto de acuerdo con su finalidad. Posee también relevancia a la hora de interpretar el texto constitucional. Sin perjuicio de que cada precepto constitucional puede tener su finalidad, hay un sistema constitucional de valores y principios que determinan los fines de la Constitución y del resto del ordenamiento. El Tribunal Constitucional español, siguiendo en este punto las pautas previamente apuntadas por el alemán, ha afirmado que el sistema constitucional de valores impone una interpretación finalista de la Constitución. ${ }^{16}$ Alguno de los criterios más utilizados en la interpretación constitucional, como el de interpretación más favorable al ejercicio de derechos fundamentales, posee un fundamento teleológico, pues implica la interpretación de las normas, acorde con la finalidad derivada de los preceptos constitucionales que recogen estos derechos fundamentales, valores y fines esenciales del sistema constitucional. En definitiva, este criterio influye o condiciona a casi todos los demás, en un

\footnotetext{
de la prerrogativa), ha destacado la relevancia y significación de los valores incorporados a los artículos 10 y 15 de la Constitución, basándose en su propia colocación en el texto constitucional, al inicio del título I y de la sección $1^{\text {a }}$ del capitulo II del mismo (STc 53/1985, de 11 de abril, f. j. 3). En general, ha afirmado reiteradamente que debe realizarse una interpretación sistemática de la Constitución, ya que la misma "es un todo en el que cada precepto adquiere su pleno valor y sentido en función del conjunto" (sstc 101/1983, de 18 de noviembre, f. j. 3; 67/1.984, de 7 de junio, f. j. 2, entre otras). De ahí la relación entre el argumento sistemático y el principio de unidad de la Constitución. Pero debe además destacarse la especial trascendencia que posee el argumento sistemático, no ya en la interpretación del propio texto constitucional, sino también en la interpretación de la ley y del resto del ordenamiento. Éste debe interpretarse, siempre que sea posible, de conformidad con la norma fundamental, cúspide del ordenamiento y que impone asi una interpretación coherente del mismo, dotando de cierta unidad a toda la interpretación jurídica. El Tribunal Constitucional ha reiterado esta necesidad, en tanto sea posible, de interpretar el ordenamiento de conformidad a la Constitución (entre otras muchas, sstc 19/1982, de 5 de mayo, f. j. 7; 75/1982, de 13 de diciembre, f. j. 2; 122/1983, de 16 de diciembre, f. j. 1, 5, 6 y 7; 93/1.984, de 16 de octubre, f. j. 5; 105/1987, de 22 de junio, f. j. 2). Sobre éste y otros argumentos utilizados por el Tribunal Constitucional español, es de gran interés el trabajo de Ezouiaga Ganuzas, Francisco Javier. La argumentación en la justicia constitucional española, Ivap, Oñati, 1987 pp. 93.

${ }^{15}$ STC $5 / 1981$, de 13 de febrero, f. j. 9, y otras posteriores.

${ }^{16}$ STC $18 / 1981$, de 8 de de junio, f. j. 2.
} 
sistema constitucional que sitúa a determinados valores como fines esenciales de todo el ordenamiento. Por ello, apuntábamos que los métodos tradicionales poseen sus propias peculiaridades cuando se utilizan en la interpretación constitucional. En todo caso, este criterio plantea también algunos problemas. En ocasiones, es preciso determinar previamente el significado de esos grandes valores y principios que actúan como fines de la Constitución y del ordenamiento. En cierto sentido, determinar el espíritu y finalidad de una norma es muchas veces un objetivo de la interpretación, más que un instrumento o criterio para la misma.

La “interpretación evolutiva”. Aparte de estos criterios, argumentos o elementos tradicionales (y de otros que podrían señalarse, como la analogía, el argumento a contrario o a fortiori, o el argumento de reducción al absurdo), hay que referirse también a la utilización del llamado criterio evolutivo. El Código Civil español se refiere en su artículo 3.1 a la realidad social del tiempo en que han de ser aplicadas las normas. Pero este elemento posee mayor trascendencia en la interpretación constitucional, dado que la Constitución ha de tener una especial pretensión de permanencia y estabilidad. De este modo, resulta la norma fundamental y suprema de un sistema político y social llamada a perdurar en el tiempo. Esta permanencia no sería posible si la interpretación de la misma no se realizase tomando en cuenta la realidad social a la que ha de aplicarse. Por ello, se ha destacado la importancia de una interpretación evolutiva del texto normativo supremo, que lo vaya adecuando a las cambiantes circunstancias sociales, políticas, económicas, sin llegar a tergiversar o ignorar el significado literal de sus preceptos.

De hecho, esa interpretación evolutiva, junto con otros factores, permite entender la larga pervivencia de algunos textos constitucionales, como el de Estados Unidos. ${ }^{17}$ El Tribunal Constitucional español ha enfatizado la importancia de este criterio. Asimismo, sigue la idea de la Corte Suprema de Canadá sobre la Constitución como "árbol vivo" y reclama una lectura evolutiva de la Constitución. Esto con base en la noción de cultura jurídica, la cual:

no se construye sólo desde la interpretación literal, sistemática u originalista de los textos jurídicos, sino que también contribuyen a su configuración la observación de la realidad social jurídicamente relevante, sin que esto signifique otorgar fuerza normativa directa a lo fáctico, las opiniones de la doctrina jurídica y de los órganos consultivos previstos en el propio ordenamiento, el derecho comparado que se da

\footnotetext{
${ }^{17}$ Wolfe, Christopher. La transformación de la interpretación constitucional, Civitas, Madrid, 1986.
} 
en un entorno socio-cultural próximo y, en materia de la construcción de la cultura jurídica de los derechos, la actividad internacional de los Estados manifestada en los tratados internacionales, en la jurisprudencia de los órganos internacionales que los interpretan, y en las opiniones y dictámenes elaboradas por los órganos competentes del sistema de Naciones Unidas, así como por otros organismos internacionales de reconocida posición. ${ }^{18}$

Pero, aun reconociendo la utilidad, incluso la necesidad, de todos los elementos analizados a la hora de interpretar la Constitución, éstos no permiten, por sí mismos, realizar planamente la labor interpretativa de todos los preceptos constitucionales. Se trata de elementos necesarios, pero no suficientes. Además, estos elementos no configuran un método propio de la interpretación constitucional, ni responden si el método es necesario. Por ello, algunos autores, destacando las peculiaridades de la interpretación constitucional, han propuesto métodos específicos para esta labor. Los más relevantes se presentan a continuación.

En esta línea, cabe destacar a Hesse, ${ }^{19}$ quien propone un método basado en la concretización. Implica precomprensión de la norma y del problema. El proceso consistente en una actuación tópica, orientada y limitada por la norma. Mediante esta actuación y la inventio, se encuentran puntos de vista que se someterán a opiniones, a favor y en contra, para fundamentar la decisión. En este proceso, juegan un papel importante los métodos tradicionales y los principios propios de interpretación constitucional.

Bóckenförde, ${ }^{20}$ por su parte, tras realizar una crítica de otros métodos propuestos para la interpretación constitucional, señala que los mismos conducen a la degradación de la normatividad de la Constitución y su equiparación a la ley. Afirma que el método de interpretación de la Constitución ha de vincularse a la teoría de la Constitución que se defienda. Pero, en todo caso, debe partir de la característica esencial de entender la Constitución como un ordenamiento marco, que fija límites y determina directrices.

También puede destacarse el denominado método tópico, con origen en la doctrina alemana, y que entre nosotros ha defendido y explicado Pérez Royo. ${ }^{21}$ Este método parte de la especial estructura normativa de la Constitución y las peculiaridades de su relación con la ley. Ésta no es equiparable a la relación

\footnotetext{
${ }^{18}$ STC 198/2012, de 6 de noviembre, f. j. 9.

${ }^{19}$ HeSSE, KonRAD. "La interpretación constitucional", en Escritos de Derecho constitucional, Centro de Estudios Constitucionales, Madrid, 1992, p. 35.

${ }^{20}$ Böckenförde, Ernst Wolfgang. "Los métodos...", op. cit.

${ }^{21}$ Pérez Royo, Javier. Curso..., op. cit., pág. 146.
} 
entre otras fuentes de distinto rango. Desde esta perspectiva, la Constitución no determina el contenido de la ley ni la actuación del legislador, sino que fija sólo los límites de tal actuación. Ello debe tener reflejo en el método de interpretación; debe invertirse en relación con el método jurídico ordinario, partiendo del problema y de la respuesta que el mismo recibe del legislador. Desde ahí, hay que comprobar si dicha respuesta ha excedido o no los límites constitucionales. De alguna forma, el método no empieza por la Constitución, sino que concluye en ella. La interpretación constitucional es así una interpretación de límites, y el método se caracteriza por la imprecisión, que trata de paliarse con la ayuda de criterios interpretativos específicos.

Todas estas propuestas tienen el acierto de poner de relieve la especificidad de la Constitución, que además de ser la norma suprema de un ordenamiento, por su especial rigidez y textura, no puede pretender establecer una regulación acabada y global. Tampoco puede cerrar las legítimas opciones del legislador democrático, o del poder de reforma como poder constituyente constituido. De esta manera, la norma fundamental ha de configurar un orden siempre abierto, ${ }^{22}$ tanto en su desarrollo como en su revisión. La Constitución se configura así como un marco, cuya función es establecer límites y mandatos más o menos genéricos, más que establecer pautas concretas que los poderes constituidos deban limitarse a ejecutar. Son notorias las consecuencias de esta idea en la interpretación de la Constitución, por lo cual cabe afırmar que esta labor no puede realizarse sin más con los métodos de la interpretación jurídica en general.

Con todo, en las últimas décadas, el método de interpretación que más éxito parece haber tenido en la interpretación de la Constitución es el de la ponderación. No es posible en este espacio profundizar sobre el significado de este método, pero cabe apuntar los siguientes parámetros: 1) la ponderación sería un método para resolver los conflictos entre derechos, partiendo de la consideración de que los derechos son en realidad principios y no reglas, en la clasificación de Alexy;23 2) Los principios no se aplican según el criterio "o todo, o nada", sino que permiten una gradualidad en su aplicación. De este modo, los conflictos entre derechos no se resuelven jerarquizándolos o sacrificando al que quede en posición inferior o supeditada, sino estableciendo fórmulas que permitan la maximización de ambos derechos y la minimización de sus límites, precisamente en una labor de ponderación; 3) La labor de ponderación no sólo la llevan a cabo los jueces, sino también, y en primera instancia, el

\footnotetext{
${ }^{22}$ Al respecto, Diaz Revorio, Francisco Javier. La Constitución como orden abierto, McGraw Hill, Madrid, 1997.

${ }^{23}$ AleXY, RoBert. Teoría de los derechos fundamentales, traducción de Carlos Bernal Pulido, Centro de Estudios Políticos y Constitucionales, Madrid, $2^{2}$ edición, 2007. Véase también el importante y más reciente artículo de AleXY, RoBerT. "La dignidad humana y el juicio de proporcionalidad", en Parlamento y Constitución. Anuario, No. 16, 2014, p. 9.
} 
legislador. Al aprobar leyes, éste debe, expresa o implícitamente, considerar el peso de los principios en juego que pueden entrar en contradicción, si bien en esta labor el legislador tiene un amplio margen. Su labor será lícita siempre que no vulnere o desconozca ninguno de dichos principios; 4) La labor del juez constitucional, valorar la obra del legislador, consiste en comprobar si la ponderación es acorde con los principios constitucionales en juego, de tal manera que el sacrificio que cada uno de ellos pueda sufrir no resulte, en las circunstancias del caso, desproporcionado.

Es aquí donde aparece un nuevo criterio de gran relevancia, relacionado, pero diferente a la ponderación que acabamos de describir sucintamente. Se trata del principio de proporcionalidad. En síntesis, es un criterio que permite determinar si la restricción de un derecho-principio determinado es o no legítima. Es decir, cuando una afectación a un derecho se convierte en una vulneración del mismo. Para que esa restricción, normalmente como consecuencia de la intervención legislativa, sea legítima, ha de superar un triple test, que consiste en la idoneidad, necesidad y proporcionalidad de la medida ${ }^{24}$ (aunque en otros casos se utilizan otros términos como finalidad, congruencia y proporcionalidad). Además, los distintos tribunales utilizan a veces un mayor o menor nivel de intensidad en las exigencias de este test de proporcionalidad, llegándose a hablar de "test estándar", o de "escrutinio más estricto" según los casos.

En todo caso, se aprecia así que la proporcionalidad puede enmarcarse en un método de ponderación, pero hay claras diferencias entre ambas. La ponderación sirve para resolver conflictos entre principios constitucionales y sería un método o criterio; la proporcionalidad sirve más bien para valorar la constitucionalidad de leyes, normas o actos infraconstitucionales, en la medida en que afectan a derechos-principios constitucionales. El primer elemento del test (la finalidad o idoneidad) permite en ocasiones situar a otros derechos, bienes o principios constitucionales como elementos justificadores de la norma o medida restrictiva de un derecho y lo hace aproximando el planteamiento al del conflicto entre principios. No obstante, permanece la diferencia de que el principio de proporcionalidad actúa en el plano de la valoración constitucional de normas; mientras la ponderación se utiliza en el ámbito de los conflictos entre principios constitucionales.

En todo caso, el éxito de la ponderación como método interpretativo nos podría llevar a plantearnos si es este el método propio y específico de la interpretación constitucional. En realidad, se trata de un criterio para resolver

\footnotetext{
${ }^{24}$ Véase Bernal Pulido, Carlos. El principio de proporcionalidad y los derechos fundamentales, Centro de Estudios Políticos y Constitucionales, Madrid, 2007, p. 692; y González Bellfuss, Markus. El principio de proporcionalidad en la jurisprudencia del Tribunal Constitucional, Thomson-Aranzadi, Cizur Menor, 2003.
} 
conflictos entre principios, y no preceptos constitucionales en todo caso, ni la norma constitucional como un todo. Si en la Constitución predominan los principios más o menos generales, ni toda norma constitucional es un principio, ni todos los principios están en la Constitución, ni la interpretación de los principios se reduce al problema de los conflictos entre ellos. Cuando introducimos además la cuestión del rango, como en el caso de conflictos entre principios constitucionales e infraconstitucionales, la ponderación no resulta ya el único criterio para resolver el conflicto. No siempre estarán excluidos otros aspectos (como un criterio de especialidad o los argumentos interpretativos ya analizados) al utilizar la ponderación.

Por lo demás, a pesar del indiscutible éxito de la ponderación, también se han señalado en ocasiones sus problemas y sus límites. Ejemplo de esto es cierta relativización de los derechos, a donde parece conducir su aparente incompatibilidad con la idea de intangibilidad de la dignidad, o sus problemas de armonización con la del contenido esencial. ${ }^{25}$

Por todo ello, no parece poder afirmarse que exista un método propio y global para la interpretación de la Constitución. No hay una fórmula del todo, por más que los autores la busquen y la defiendan. La Constitución, a pesar de sus muchas especialidades, no deja de ser una norma. Y esas propias especialidades están a veces presentes, aunque en menor medida, en otras normas, con lo cual la cuestión de las peculiaridades debe matizarse. En primer lugar, el carácter relativamente abierto es una característica que también puede encontrarse en algunos preceptos legales. En segundo lugar, la Constitución es un marco para la ley y el legislador. Pero no es un solo marco, pues en ocasiones también contiene mandatos generales, o incluso con cierta concreción, que necesariamente deben imponerse a la ley, y de alguna manera determinan parcialmente su contenido. Esta característica parece ser frecuente en algunas de las Constituciones más recientes, o mas reformadas, distinguidas por su extensión y por el detalle de ciertas de sus regulaciones. En tercer lugar, la interpretación de la Constitución no siempre se realiza en el contexto de la valoración de la obra legislativa y su conformidad con la norma fundamental.

Cotidianamente, los tribunales ordinarios deben interpretar la Constitución para resolver casos concretos, supuestos de hecho equiparables a los que son objeto de los asuntos ordinarios (en realidad, para aplicarla a los mismos supuestos ordinarios). Incluso el Tribunal Constitucional, en algunos procesos,

\footnotetext{
${ }^{25}$ Véase Aleinikoff, Thomas Alexander. El Derecho Constitucional en la era de la ponderación, Palestra, Lima, 2010. Por otro lado, es cierto que Robert Alexy ha respondido con profundidad y rigor al problema de la compatibilidad entre la ponderación y la dignidad a través de la llamada "fórmula del peso", en su trabajo "La dignidad humana y el principio de proporcionalidad". Robert, Alexy. Op. cit.
} 
y particularmente en el amparo, no se limita a la valoración constitucional de la ley o de otras normas, sino que ha de valorar actos de los poderes públicos y a veces de particulares. A pesar de ser relevantes las peculiaridades mencionadas e incuestionable la utilidad de algunas ideas presentes en los métodos específicos, no configuran un método radicalmente diferente al propio de la interpretación jurídica en general.

En todo caso, resulta conveniente destacar algunos de los criterios o elementos específicos que se han apuntado como propios de la interpretación constitucional, y que deben estar presentes siempre en esta labor interpretativa. En primer lugar, puede apuntarse el principio de unidad de la Constitución, el cual supone considerar a ésta como un todo que se sitúa en la cúspide del ordenamiento y debe presidir, a su vez, la interpretación de éste. En segundo lugar, el principio de concordancia práctica, según el cual los conflictos posibles entre preceptos constitucionales no deben resolverse con base en la supuesta superioridad de alguno de ellos y el sacrificio de otros, o excluyendo la aplicación de alguno de ellos, sino mediante la ponderación, en cada caso concreto, que permita una cierta realización de los principios en tensión. En tercer lugar, hay que destacar el principio de fuerza normativa de la Constitución, el cual presupone el carácter jurídico y vinculante de cada uno de sus preceptos. En cuarto lugar, el principio de corrección funcional, el cual implica el respeto a la distribución de poderes y funciones derivada de la propia Constitución.

De este modo, la suma de los criterios tradicionales y de los específicos de la Constitución (literal, sistemático, histórico, teleológico, interpretación evolutiva, concordancia práctica, unidad de la Constitución, corrección funcional, carácter abierto de sus preceptos, ponderación) llevará a la utilización conjunta de todos, o algunos de ellos, según los casos. Esto permitirá resolver la mayoría de los problemas interpretativos, siempre tomando en cuenta que, en caso de duda o ambigüedad, el primer sujeto legitimado para interpretar y desarrollar la constitución es el legislador.

\section{Los intérpretes de la Constitución}

Cualquier sujeto, público o privado, puede interpretar la Constitución. Los autores que forman parte de la doctrina jurídica lo hacen habitualmente, aunque esta interpretación no tenga siempre un valor jurídico, en sentido estricto. Los poderes públicos también lo hacen de forma cotidiana, pues buena parte de sus actuaciones presuponen una determinada interpretación de los preceptos constitucionales, así como de los legales. Así también, en ocasio- 
nes, han aplicar la Constitución a supuestos concretos, ya sea directamente o con la mediación de otras normas jurídicas. Incluso puede afirmarse que los particulares efectúan una previa interpretación de la Constitución cuando realizan actuaciones con relevancia jurídica. Ello, a pesar de que buena parte de los preceptos constitucionales no vayan dirigidos directamente a ellos. Por ejemplo, cuando dos ciudadanos firman un contrato, pueden partir del presupuesto de que el mismo no vulnera derechos fundamentales de ninguno de ellos; cuando una persona se expresa a través de un medio de comunicación, puede entender que no está vulnerando el honor o la intimidad de otros. En ambos casos, esa actuación presupone una previa interpretación de la norma fundamental, aunque el criterio que los particulares mantengan sobre dicha interpretación no es decisivo ni definitivo.

Pero entre todos los sujetos que pueden interpretar la Constitución, destaca la posición de algunos, ya que sus interpretaciones alcanzan una especial relevancia, dada la función que el propio texto constitucional les confiere. En esta línea hay que apuntar al legislador, al Tribunal Constitucional, y a los jueces y tribunales integrantes del Poder Judicial. Estos últimos juegan un papel decisivo en la interpretación de todo el derecho. La Constitución es la norma suprema del ordenamiento, por lo cual también deben interpretarla y aplicarla en la resolución de las concretas controversias que se les presentan, siendo sus decisiones vinculantes para las partes del proceso.

Con todo, merece especial comentario la posición de los otros dos intérpretes cualificados de la Constitución. En primer lugar, el legislador, que es el intérprete primario de la norma fundamental. Conviene aclarar esta afırmación. Desde luego, la función esencial del legislador no es la interpretación de la Constitución, sino la aprobación, en representación de la voluntad popular, de normas jurídicas generales denominadas leyes que tienen el rango superior en el sistema de fuentes (con la excepción de la propia Constitución). Esta función legislativa no se ejerce para interpretar la Constitución; en la mayoría de las ocasiones, no se ejerce siquiera con la intención de desarrollarla, sino simplemente con la finalidad de dar respuesta normativa a distintas situaciones o problemas de la sociedad. Pero el ejercicio de esta función sí presupone una previa interpretación de la Constitución. La norma suprema contiene los límites que debe respetar toda actuación legislativa, los principios que ha de acoger esta actuación, así como, en ocasiones, mandatos concretos dirigidos al legislador. De tal forma, no es posible esta actuación sin una previa interpretación de la Constitución, aunque a veces ésta sea meramente implícita. Por ello, puede decirse que el legislador es el intérprete primario y, en cierto sentido, "cotidiano" de la Constitución. 
Sin embargo, lo anterior no le permite situar su interpretación en el mismo rango que la norma interpretada. Una cierta línea de la jurisprudencia constitucional española, no exenta de críticas, ha afirmado que el legislador no puede precisar "el único sentido, entre los varios posibles, que deba atribuirse a un determinado concepto o precepto de la Constitución, pues al reducir las distintas posibilidades o alternativas del texto constitucional a una sola, completa de hecho la obra del poder constituyente y se sitúa funcionalmente en su plano", es decir, no puede aprobar leyes "meramente interpretativas". ${ }^{26}$ Por supuesto, esta jurisprudencia no afecta a la idea de que toda legislación presupone una previa interpretación, sino simplemente impide que el precepto legal pretenda señalar como único sentido una entre las posibles interpretaciones alternativas de la Constitución. Con todo, las afirmaciones del Tribunal Constitucional plantean no pocas dudas y dificultades. Si un precepto legal realiza de forma expresa una interpretación de la Constitución, incluso descartando otras posibles, no supone que esa interpretación se "incorpore" a la norma fundamental ni adquiera su rango, ya que puede ser sustituida por cualquier otra ley posterior.

Nadie hay más cualificado o mejor situado que el legislador para elegir entre las diversas posibilidades o alternativas del texto constitucional. La vinculación de ese precepto legal "meramente interpretativo" a otros poderes y a los ciudadanos deriva del propio sometimiento a la ley. Desde luego, dicha interpretación legislativa no vincula al Tribunal Constitucional que, en su caso, puede declarar su inconstitucionalidad. Otra cosa es que la interpretación realizada por el legislador sea contradictoria con el propio precepto constitucional, o no tenga cabida dentro de los límites de un concepto constitucional, aunque éste sea parcialmente abierto. Así, por ejemplo, y con mejor criterio, el Tribunal Constitucional español señaló que cierta interpretación del concepto de "flagrante delito" a que alude el artículo 18.2 de la Constitución, realizada por un precepto legal, era inconstitucional. Pero no por interpretar dicho concepto, sino porque la interpretación elegida contradice el propio sentido y significado constitucional del mismo. ${ }^{27}$

También el Tribunal Constitucional es otro de los intérpretes destacados de la Constitución. En comparación con el legislador, sería un intérprete "secundario", en tanto su interpretación sólo se produce en caso de conflictos constitucionales, y a instancia de parte legitimada. Esto implica que se ha puesto en cuestión una interpretación previa de la norma fundamental, ya sea (en los supuestos de control de constitucionalidad) la realizada por el legislador,

\footnotetext{
${ }^{26}$ Sstc 76/1.83, de 5 de agosto, f. j. 4; 227/1988, de 29 de noviembre, f. j. 3; 17/1991, de 31 de enero, f. j. 7, entre otras. ${ }^{27}$ STC 341/1993, de 18 de noviembre, f. j. 8.
} 
ya sea la que han realizado otros poderes u órganos públicos, y en particular los órganos jurisdiccionales. A pesar de ese carácter secundario, se considera acertadamente que el Tribunal Constitucional es el intérprete supremo de la Constitución.

\section{El tribunal constitucional y la interpretación de la Constitución}

En cualquier sistema donde exista una garantía jurisdiccional de la supremacía constitucional, puede afirmarse que todo juez es juez constitucional. Con independencia del modelo de justicia constitucional, más o menos concentrado, todo juez ha de participar en la función de interpretar y aplicar la Constitución en todo tipo de procesos. Es posible, incluso, que no exista un Tribunal Constitucional o una jurisdicción constitucional específica, ya que todas las funciones de garantía constitucional pueden recaer en el Poder Judicial (así sucede en los llamados modelos difusos). Pero, por muy centralizado que sea el modelo, el monopolio del Tribunal Constitucional ( $u$ órgano en el que se concentran las correspondientes funciones, que podría ser una sala constitucional en una Corte Suprema) llegará como mucho a la posibilidad de no aplicar o derogar las leyes inconstitucionales. Esta función, en los modelos mixtos que son mayoría en Iberoamérica, comparte de todos modos con los tribunales ordinarios. Pero, en ningún caso, ni en el más centralizado de los modelos, los tribunales ordinarios pierden su esencial función de interpretar y aplicar la Constitución, así como interpretar las leyes de conformidad con la Constitución. Esta última función conlleva cierto efecto de depuración del ordenamiento o de control de constitucionalidad. Elegir una interpretación entre varias posibles implica descartar y no aplicar otras interpretaciones, las cuales serían normas derivadas alternativamente de la misma disposición legal, que se consideran inconstitucionales. ${ }^{28}$

Por tanto, lo que distingue al Tribunal Constitucional ( $u$ órganos equivalentes de una jurisdicción específicamente constitucional) en su papel de garante de la supremacía constitucional, respecto a los tribunales ordinarios, es que tiene la última palabra. Por ello, en varios ordenamientos, la Constitución o las leyes califican al Tribunal Constitucional como supremo intérprete de la Constitución. A partir de ahí, el reparto de funciones en la materia, entre Tribunal Constitucional y tribunales ordinarios, dependerá del modelo de control constitucional, en el que hoy la distinción "control difuso-control concentrado" no permite explicar la gran variedad de posibilidades que existe. ${ }^{29}$ Pero sí puede

\footnotetext{
${ }^{28}$ Véase Diaz Revorio, Francisco Javier. Las sentencias interpretativas del Tribunal Constitucional, Porrúa, México, 2011.

${ }^{29}$ Véase Fernández Segado, Francisco. "La obsolescencia de la bipolaridad 'modelo americano-modelo europeokelseniano' como criterio analítico del control de constitucionalidad y la búsqueda de una nueva tipología
} 
afirmarse con carácter general que la función de garantía jurisdiccional de la Constitución es compartida y que el Tribunal Constitucional, allí donde existe, tiene la última palabra. En su defecto, suele ser un órgano de la propia justicia ordinaria el que desempeña ese papel, como la Corte Suprema en el modelo de los Estados Unidos, que a pesar de ser difuso está basado en el principio stare decisis, lo que introduce un elemento de gran uniformidad.

De este modo, como intérprete supremo de la Constitución, el Tribunal es constitucionalmente infalible, pues ningún otro órgano puede corregir o rectificar sus decisiones. Trasladando las célebres afirmaciones del juez Jackson referidas al Tribunal Supremo norteamericano, we are not final because we are infallible, but only we are infallible because we are final. Puede parecer difícil de justificar este poder "infalible", pero la experiencia histórica ha demostrado que sólo un sistema que contemple el control jurisdiccional de la adecuación de los poderes del Estado a la Constitución, sólo un modelo de justicia constitucional, garantiza eficazmente la supremacía de la Constitución sobre el resto del ordenamiento y, en suma, el propio carácter jurídico de la norma fundamental. Por lo demás, la actuación del Tribunal Constitucional no carece totalmente de límites:

a) En primer lugar, al actuar en cierto modo como "comisionado del poder constituyente”, está sometido a éste, que puede modificar la Constitución alterando su configuración y funciones, o incluso suprimiéndolo.

b) En segundo lugar, el Poder Legislativo posee, dentro del respeto a la Constitución, amplias facultades en relación con el Tribunal Constitucional, al poder aprobar o modificar su legislación.

c) En tercer lugar, el mismo Poder Legislativo, eventualmente, con participación de otros órganos o poderes del Estado, designa a los miembros del Tribunal Constitucional.

d) En cuarto lugar, en lo relativo al ámbito concreto de la garantía de los derechos, el Tribunal Constitucional aparece en la práctica "sometido" al superior criterio de tribunales internacionales, como el Tribunal Europeo de Derechos Humanos o la Corte Interamericana de Derechos Humanos, ya que en buena medida los derechos protegidos en las constituciones nacionales coinciden con los que recogen los correspondientes convenios de ámbito regional. 
Con todo, ha de reconocerse que el correcto funcionamiento del Tribunal Constitucional, y su actuación dentro de sus límites constitucionales, pasan también por ciertas dosis de self restraint. Asimismo, la legitimidad de su actuación dependerá de que sea capaz de demostrar, con una correcta motivación reflejada en la argumentación jurídica, que sus decisiones suponen la más correcta aplicación de la Constitución al caso concreto, o al menos una adecuada aplicación de la norma fundamental, sin separación, abandono o quebrantamiento de la misma.

\section{El valor de la jurisprudencia constitucional}

Si la interpretación llevada a cabo por el Tribunal Constitucional como intérprete supremo prevalece sobre la realizada por cualquier otro órgano o poder, las decisiones que contienen esa interpretación poseen un especial valor en el sistema jurídico. En un sentido amplio, estas decisiones configuran lo que suele denominarse jurisprudencia constitucional, pero el análisis de este concepto requiere mayor precisión. Aunque en un primer sentido ésta podría definirse por su objeto, toda la jurisprudencia que contiene la interpretación de la Constitución o la interpretación constitucional de la ley, en un sentido más estricto se referiría sólo a la que procede del Tribunal Constitucional.

Aun así, la expresión todavía permite varios sentidos. En sentido amplio, haría referencia a la doctrina contenida en todas sus resoluciones. En efecto, el Tribunal emite resoluciones como providencias, autos y sentencias, sin que pueda equipararse el valor de todas ellas. Tampoco puede equipararse el valor de todas las partes de la resolución, siendo relevantes a los efectos que nos interesan los fallos y su fundamentación, es decir, la ratio decidendi. Además, el Tribunal no sólo interpreta la Constitución, sino también la ley. En fin, las constituciones y legislaciones nacionales suelen distinguir, entre todas las decisiones, los especiales efectos de las sentencias recaídas en procesos de inconstitucionalidad.

De este modo, cobran especial relevancia las sentencias del Tribunal Constitucional como fuente fundamental de su jurisprudencia, en tanto estas decisiones son las únicas que ponen fin al proceso. Pueden decidir (y así sucede normalmente) sobre el fondo del asunto, constituyendo las resoluciones en las que se contiene así la mayor y más relevante parte de la doctrina sustantiva.

Es imposible llevar a cabo un examen general y abstracto sobre los efectos de las sentencias del Tribunal Constitucional, ya que los mismos pueden ser diferentes, dependiendo de cada sistema y de cada ordenamiento. En este trabajo, interesa destacar el especial valor de la interpretación constitucional (y de la interpretación constitucional de la ley) llevada a cabo por el Tribunal 
Constitucional en sus sentencias, y en especial en las recaídas en procesos de control de constitucionalidad.

Siguiendo el modelo kelseniano, muchos sistemas otorgan a estas sentencias efectos derogatorios, cuando declaran la inconstitucionalidad de la ley. Ello implica la expulsión del ordenamiento, y por tanto, efectos erga omnes. Pero la interpretación de la Constitución y de la ley no sólo se contiene en estas sentencias estimatorias, sino que, incluso más frecuentemente, aparece en sentencias desestimatorias o en tipos intermedios de sentencias, especialmente en las llamadas sentencias interpretativas. En estos casos, los distintos ordenamientos prevén diferentes consecuencias para estas decisiones, aunque en muchos casos éstas no quedan claramente precisadas en la Constitución y en la ley. En todo caso, allí donde el Tribunal Constitucional es el intérprete supremo de la Constitución, esa interpretación suele tener igualmente efectos erga omnes, o bien efectos vinculantes para todos los jueces y tribunales. Ambas categorías de efectos se aproximan enormemente en la práctica, porque de la vinculación a jueces y tribunales deriva la vinculación de los demás poderes, e incluso de los ciudadanos, quienes están sometidos a las decisiones judiciales en caso de conflicto.

De hecho, constituir material jurídico vinculante para todos los jueces y tribunales convierte realmente a estas decisiones en fuente del derecho. Como ha apuntado Bocanegra, ${ }^{30}$ en nada beneficia a un ciudadano no estar vinculado a ciertas decisiones del Tribunal Constitucional, si los demás tribunales y autoridades están obligados a acatarlas.

De todo lo anterior creo que se deduce con claridad que la jurisprudencia constitucional, entendida como la interpretación de la Constitución y del resto del ordenamiento contenida en la fundamentación de todas las sentencias y autos que deciden procesos constitucionales, especialmente en los de control de constitucionalidad, es fuente del derecho. Es una de las formas en que se plasma el ordenamiento jurídico, al modificar e innovar en las normas jurídicas preexistentes. La jurisprudencia constitucional conforma así "material jurídico" al cual se vinculan todos los jueces y tribunales, al resolver las concretas controversias que se les plantean. En consecuencia, se vinculan todos a su cumplimiento.

A partir de aquí, queda por resolver la cuestión de cuál es la posición que ocupa esta jurisprudencia en el sistema de fuentes. En primer lugar, conviene precisar que la jurisprudencia constitucional ocupa una posición superior a

\footnotetext{
30 Bocanegra Sierra, Raúl. El valor de las sentencias del Tribunal Constitucional, Instituto de Estudios de la Administración Local, Madrid, 1982, pp. 70-71.
} 
la de los tribunales ordinarios, incluido el Tribunal Supremo. Esta afirmación suele ser de algún modo aplicable a aquellos sistemas en los cuales la posición de intérprete supremo recaiga en una sala constitucional en la propia Corte Suprema. Ello porque, como ya he apuntado, los juzgados y tribunales (incluido el Tribunal Supremo) están obligados a interpretar la Constitución, las leyes y los reglamentos de conformidad con lo establecido en la jurisprudencia constitucional.

Para precisar la posición de la jurisprudencia constitucional en el sistema de fuentes, es necesario distinguir según el rango de la norma interpretada 0 anulada. Si lo que da valor vinculante a la jurisprudencia constitucional es su capacidad para innovar o suprimir el ordenamiento, mediante la declaración de inconstitucionalidad de normas, o bien mediante la interpretación de las mismas, la jurisprudencia viene a incorporarse al ordenamiento con el rango de la norma interpretada o expulsada del mismo. Así, cuando la jurisprudencia interpreta la propia Constitución (desde luego, el Tribunal no puede en modo alguno anular ésta), adquiere prácticamente un rango constitucional, integrándose de alguna manera en la norma fundamental. En todo caso, se mantendría una subordinación de la jurisprudencia a la propia Constitución, que justifica y legitima toda actuación del propio Tribunal Constitucional.

Los controles para asegurar esta adecuación no actúan de una manera cotidiana y ordinaria. En cambio, cuando la jurisprudencia interpreta preceptos legales o los declara total o parcialmente inconstitucionales, adquiere valor de ley en cuanto esa jurisprudencia complementa o suprime parcialmente la ley. En lo sucesivo, el legislador puede de nuevo modificar esa ley, si no fue declarada totalmente nula, o aprobar una nueva, lo cual naturalmente afectaría a la jurisprudencia constitucional recaída sobre ella, sin perjuicio de la posibilidad de ulteriores pronunciamientos del Tribunal. En fin, en los supuestos en los que el Tribunal interpreta o declara inconstitucionales reglamentos, su jurisprudencia tendrá el valor de éstos, ya que esa jurisprudencia no afecta en modo alguno a la ley. En definitiva, la interpretación llevada a cabo por el Tribunal tendrá los mismos efectos jurídicos y vinculantes que tenía el propio reglamento. 